Article

\title{
Design of a High-Performance Digital Slit-Lamp Microscope with Five-Switched Zoom
}

\author{
Youping Huang ${ }^{1}$, Xiaogang Chen ${ }^{1, *}$, Hao Zhang ${ }^{1, *}$, Shuyan Huang ${ }^{1}$ and Feng Lin ${ }^{2}$ \\ 1 Laboratory for Advanced Sensing Technology, Fujian Jiangxia University, Fuzhou 350108, China; \\ hyp@fjjxu.edu.cn (Y.H.); hsy@fjjxu.edu.cn (S.H.) \\ 2 Fujian Provincial Key Laboratory for Photonics Technology, Fujian Normal University, \\ Fuzhou 350007, China; lfeng127@163.com \\ * Correspondence: xgchen@fjjxu.edu.cn (X.C.); 1698091502181@fjjxu.edu.cn (H.Z.)
}

Received: 23 March 2020; Accepted: 13 April 2020; Published: 16 April 2020

check for updates

Featured Application: A digital slit-lamp microscope (DSLM) with five discrete zoom functions can be a fundamental tool in ophthalmological practice. It can be used to observe the structures of the eye anterior end and diagnose various eye diseases, such as cataract, corneal injury, and retinal detachment. Combining the interface design of a CCD system, it is convenient to obtain high-quality fundus images for remote diagnosis.

\begin{abstract}
In this study, we design and present a five-fold digital slit-lamp microscope (DSLM) with built-in photographic lens and CCD. The initial structures of the front objective, Galilean telescope system, and photographic lens are systematically investigated and discussed in the design. A progressive optimization process is employed in the non-coaxial system design after the coaxial system achieves high performance. The analysis of spot diagrams and the modulation transfer function (MTF) show that this DSLM optical system achieves quasi-diffraction-limited performance and enables high-quality imaging for ophthalmic examination. Furthermore, tolerance analysis of this optical system is also performed, which provides a theoretical basis for machining and assembly. This design provides an idea for the design of a digital-zoom microscope in biomedical imaging instruments.
\end{abstract}

Keywords: optical design; digital slit-lamp microscope; non-coaxial system; tolerance analysis

\section{Introduction}

As one of the most advanced analysis instruments in biomedical science, the microscope has been rapidly developed in recent years [1-3]. Among all microscopes, the slit-lamp microscope (SLM), invented by Allvar Cullstrand, is most useful in clinical ophthalmology [4,5]. It can be used to observe the structures of the eye anterior end and diagnose various eye diseases, such as cataract, corneal injury, and retinal detachment [6-9]. The traditional SLM relies only on visual observation and needs manual mapping to record the signs of disease, which is inconvenient and unreliable. Nowadays, with the advent of cameras and the progress of digital technology, the digital slit-lamp microscope (DSLM) is favored by researchers in the field of ophthalmology due to the unique advantages of high resolution, quick acquisition, and storage of image information [10].

DSLMs contribute to transmitting images for remote diagnosis. In order to quickly and accurately determine the signs of disease, capturing high-quality and high-resolution images is essential in telemedicine consultation. The main design of a conventional SLM does not include an image acquisition module. The digital image is obtained from the SLM using a camera or smartphone by means of an adapter in the eyepiece [11-14]. C. Yen et al. reported a method of image acquisition from 
a smartphone with an SLM via a simple adapter in the eyepiece [13]. A common disadvantage of these adapters is that they need to be repeatedly mounted and dismounted, and this is inconvenient during serial observations. These images obtained by an external device lose their diagnostic quality due to the lack of correction of optical aberrations and noise introduced by the adapter system. Hence, a built-in photographic lens and CCD design can improve the image quality of photography without camera adapters. The designed DSLM can connect directly to a computer via universal serial bus (USB) and quickly obtain diagnostic-quality images from the SLM, thereby reducing the time and effort required for preparing devices, particularly when making multiple consecutive observations or when the targets disappear quickly.

In this study, we present and analyze design ideas for a high-performance DSLM. Based on the parallel optical path principle in stereoscopic microscopes, a five-switched DSLM with built-in photographic lens and CCD was designed using Zemax optical design software (Zemax, LLC, Kirkland, WA, USA). The microscope constituted a front objective, Galilean telescope system, and photographic lens; each of these was systematically investigated and discussed in the design. It is worth noting that the front objective part is non-coaxial with the other parts. In order to reduce the influence of decentration (non-coaxial), a progressive optimization process of gradual decentration correction was adopted when the coaxial system achieved high performance. The designed DSLM can realize five magnifications of $6 \times, 10 \times, 16 \times, 25 \times$, and $40 \times$ and obtain high-performance images directly without an adapter. Loose manufacturing and assembly tolerances are also provided for the design, and the system with loose tolerances can be manufactured with higher yield and lower cost. Our design with spherical surfaces has significant advantages, including low cost, easy assembly, and high-quality imaging, all of which make it attractive to clinical medicine in optometry.

\section{Design of the DSLM Optical System}

\subsection{Design Principle}

The DSLM optical system is composed of two parallel optical paths which are identical and correspond to the left and right eyes. The two parallel optical paths have a common front objective, and a schematic illustration is shown in Figure 1. The received light from the object is collected by the front objective and sequentially delivered to the Galilean telescope. Next, the incident light is divided into two beams by a beam splitter. One light is delivered to a tube lens and eyepiece, and the other is sent to a built-in photographic lens and CCD for imaging. Since a Galilean telescope can provide two magnifications, direct and inverted, magnifications of $6 \times, 10 \times, 25 \times$, and $40 \times$ can be achieved with two Galilean telescopes. A $16 \times$ magnification is provided by removing the Galilean telescopes (empty). Switching of telescopes is done by rotating the drum. In other words, five magnifications can be realized in the design.

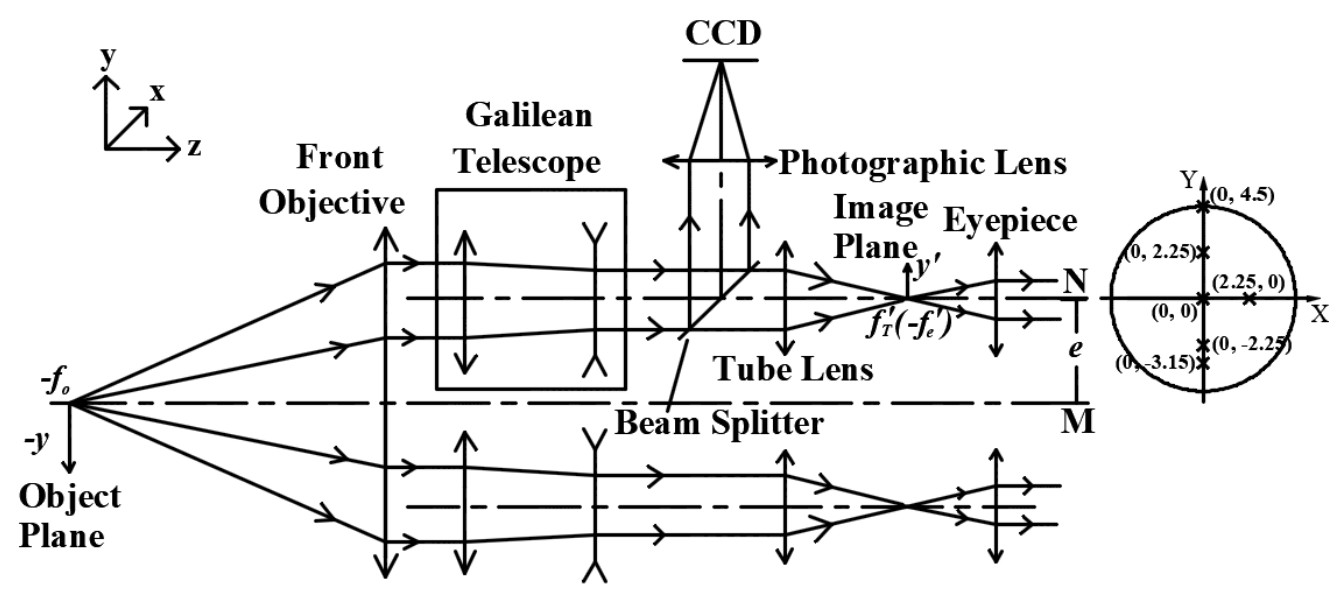

Figure 1. Optical principle of the digital slit-lamp microscope (DSLM). 
Notably, the DSLM is a non-coaxial (decentered) optical system. As shown in Figure 1, the optical axis of the front objective is assumed to be on the dash-dotted line denoted $\mathrm{M}$, and the optical axis of the Galilean telescope, beam splitter, tube lens, and eyepiece are on the other dash-dotted line, denoted $\mathrm{N}$. The optical axes $\mathrm{M}$ and $\mathrm{N}$ are parallel to each other and spaced with longitudinal distance $e$. It is well known that the aberrations of a non-coaxial optical system are the sum of the individual surface contributions. However, the forms of the aberrations have changed and become more complex, which increases the difficulty of aberration correction [15-18]. According to the calculation in [18], additional aberrations will be introduced due to the decentration (non-coaxial), which increase on the original basis with no new types of aberrations. This means that the additional aberrations in a non-coaxial system can be minimized when the aberrations are corrected to their minimum in the coaxial system. In addition, a small amount of decentration has little impact on the aberrations of the optical system on the premise that the primary aberration is small before decentration. In this case, the designed non-coaxial optical system can transition from the coaxial design, and the amount of decentration is increased gradually. An optimization process is employed in the designed non-coaxial system after the coaxial system achieves high performance. The optical system of the DSLM is divided into three subsystems: the front objective, Galilean telescope, and photographic lens. First of all, the subsystems are designed optimally based on the coaxial system. Then, the vertical position of the front objective has a tiny displacement offset from the optical axis (forming a non-coaxial axis). Optimization of the design is performed again in the non-coaxial system. The progressive optimization process should be repeatedly conducted until the performance of the design meets requirements [19-21].

\subsection{Design Specifications and Initial Structure}

As mentioned earlier, the optical system of the DSLM is divided into three subsystems: the front objective, Galilean telescope, and photographic lens. In this section, we analyze and calculate the design specifications and initial structures of these three parts. Their derivation is described as follows.

The total visual magnification $\left(M_{T O T}\right)$ of the system can be expressed as [22]

$$
M_{T O T}=\left(-{f^{\prime}}_{T} / f^{\prime}{ }_{F O}\right) \times \Gamma_{T E L} \times M_{E}
$$

where $\Gamma_{T E L}$ is the magnification of the Galilean telescope. The quantity $M_{E}$ is the magnification of the eyepiece, which is set as $12.5 \times$. The quantities $f^{\prime}{ }_{F O}$ and $f^{\prime}{ }_{T}$ are the focal lengths of the front objective and tube lens, respectively. As described previously, when $M_{T O T}=16 \times, \Gamma_{T E L}=1 \times$ (the Galilean telescopes are empty), so $\left(-f^{\prime}{ }_{T} / f^{\prime}{ }_{F O}\right)=1.28$. It can be deduced that the corresponding magnifications of the Galilean telescope $\left(\Gamma_{T E L}\right)$ are $0.4 \times, 0.625 \times, 1.6 \times$, and $2.5 \times$, while the total visual magnifications $\left(M_{T O T}\right)$ are $6 \times, 10 \times, 25 \times$, and $40 \times$, respectively. Note that in the front objective, the field of view (FOV) and f-number decrease as the magnification increases [23]. An FOV of $6 \times$ and f-number of $40 \times$ should be taken as the initial specifications of the front objective. Generally, the aperture diameter $\left(D_{F O}\right)$ of the front objective is no less than $40 \mathrm{~mm}$ at $40 \times$ magnification (set to $40 \mathrm{~mm}$ for $D_{F O}$ ). For convenient detection and treatment, a large working distance of the optical system is necessary, so we set the focal length of the front objective $\left(f_{F O}^{\prime}\right)$ to $110 \mathrm{~mm}$. Hence, the f-number is ${f^{\prime}}_{F O} / D_{F O}=2.75$ for the front objective. The FOV can be obtained by [22]

$$
\begin{gathered}
\beta=\left(-{f^{\prime}}_{T} / f^{\prime}{ }_{F O}\right) \times \Gamma_{T E L}=y^{\prime} / y, \\
\tan \omega_{F O}=y / f_{F O}^{\prime} .
\end{gathered}
$$

where $\beta$ is the vertical magnification of the combined microscope objective. $\Gamma_{T E L}$ of $0.4 \times$ was selected for Equation (2). In this design, the field number (F. N.) of view, defined as the diameter of the bright circle which can be seen in the eyepiece, was set to $20 \mathrm{~mm}$. The corresponding image height $\left(y^{\prime}\right)$ of the microscope objective is $10 \mathrm{~mm}$ in practical applications. Therefore, the FOV, $2 \omega_{F O}=20.13^{\circ}$, can be calculated for the front objective. 
Two Galilean telescopes are installed in the rotating drum and used to provide four magnification rates in the system. The distance between the objective and eyepiece of the Galilean telescope was taken to be $30 \mathrm{~mm}$. Therefore,

$$
f^{\prime}{ }_{1}-f_{2}=30,
$$

where ${f^{\prime}}_{1}\left(f_{1}\right)$ and ${f^{\prime}}_{2}\left(f_{2}\right)$ are the focal lengths of the objective and eyepiece of the Galilean telescope. Also,

$$
\Gamma_{T E L}=-f^{\prime}{ }_{1} / f^{\prime}{ }_{2} .
$$

We can calculate the focal lengths, $f^{\prime}{ }_{1}$ and ${f^{\prime}}_{2}$, according to Equations (4) and (5) when $\Gamma_{T E L}=$ $1.6 \times$ and $2.5 \times$. The optical path from the front objective is shared by two identical Galilean telescopes, so the maximum aperture diameter of the Galilean telescope objective was set to $D_{T E L}=D_{F O} / 2=20$ $\mathrm{mm}$. The f-number of the objective of the Galilean telescope can be obtained by calculating the ratio of $f^{\prime}{ }_{1}$ to $D_{T E L}$.

The half FOV of the objective $\left(\omega_{T E L}\right)$ and eyepiece $\left(\omega^{\prime} T E L\right)$ of the Galilean telescope can be calculated by

$$
\tan \omega_{T E L}=\tan \omega^{\prime} T E L / \Gamma_{T E L}
$$

The parallel light entering the Galilean telescope objective is transmitted from the front objective, which indicates that the FOV of the objective of the Galilean telescope is equal to the FOV of the front objective $\left(\omega_{T E L}=\omega_{F O}\right) . \omega_{F O}$ can be calculated using Equation (3) with different magnifications. Finally, $\omega_{T E L}$ and $\omega^{\prime} T E L$ can be obtained by calculation using Equations (3) and (6).

The CCD is preset for imaging in the DSLM. This sensor is a $1 / 1.8^{\prime \prime}$ type CCD $(7.2 \times 5.3 \mathrm{~mm})$ which packs 8.5 million total pixels and has a pixel pitch of just $2.2 \mu \mathrm{m}$. In order to make sure that the same image is observed by the eyepiece and CCD, the focal length of the photographic lens $\left(f^{\prime}{ }_{P H O}\right)$ should satisfy the following equation:

$$
l /\left(\frac{f^{\prime}{ }_{P H O}}{f^{\prime}{ }_{0}} \times \Gamma_{T E L}\right)=2 y^{\prime} /\left(\frac{f^{\prime}{ }_{T}}{f^{\prime}{ }_{0}} \times \Gamma_{T E L}\right),
$$

where $l$ is the wide side of the CCD target surface $(l=7.2 \mathrm{~mm})$. Thus, the f-number $\left(f^{\prime}{ }_{P H O} / D_{P H O}\right)$ and half FOV $\left(\omega_{\mathrm{PHO}}\right)$ of the photographic lens can be defined as [22]

$$
\begin{gathered}
N_{C C D}=\frac{1}{\sigma^{\prime}} \\
N_{L}=\frac{D_{P H O}}{1.22 \lambda f^{\prime}{ }_{P H O}}, \\
\tan \omega_{P H O}=h_{C C D} / f^{\prime}{ }_{P H O},
\end{gathered}
$$

where $\sigma$ is the pixel size of the CCD $(\sigma=2.2 \mu \mathrm{m})$ and the wavelength $\lambda$ is set to $550 \mathrm{~nm}$. The resolution of the system depends on the resolution of the photographic lens and CCD. As the Buckets effect reveals, the best match makes the resolutions of the photographic lens and CCD equal $\left(N_{\mathrm{CCD}}=N_{\mathrm{L}}\right)$. The quantity $h_{C C D}$ is the half-diagonal of the CCD image size $(7.2 \times 5.3 \mathrm{~mm})$, so $h_{C C D}=0.5 \times\left(7.22^{2}+\right.$ $\left.5.32^{2}\right)^{1 / 2}$.

Thus far, all design specifications of the front objective, Galilean telescopes, and photographic lens have been taken into account and calculated. The overall specifications of the system are summarized in Table 1. 
Table 1. Design specifications of the DSLM.

\begin{tabular}{|c|c|c|c|c|c|}
\hline \multicolumn{6}{|c|}{ Basic Specifications } \\
\hline \multirow{2}{*}{\multicolumn{2}{|c|}{$\begin{array}{l}\text { Magnification of eyepiece } \\
\text { Image sensor type }\end{array}$}} & $12.5 \times$ & \multicolumn{2}{|c|}{$\begin{array}{l}\text { Field Number } \\
\end{array}$} & $20 \mathrm{~mm}$ \\
\hline & & $1 / 1.8^{\prime \prime} \mathrm{CCD}$ & \multicolumn{2}{|c|}{ Design spectrum } & Visible light \\
\hline \multicolumn{3}{|c|}{ Total visual magnification } & \multicolumn{3}{|c|}{$6 \times, 10 \times, 16 \times, 25 \times, 40 \times$} \\
\hline \multicolumn{3}{|c|}{ Front objective } & \multicolumn{3}{|c|}{ Photographic lens } \\
\hline \multicolumn{2}{|c|}{ Focal length (mm) } & 110 & \multicolumn{2}{|c|}{ Focal length (mm) } & 50.7 \\
\hline \multicolumn{2}{|c|}{ F-number } & 2.75 & \multicolumn{2}{|c|}{ F-number } & 3.28 \\
\hline \multirow{2}{*}{\multicolumn{3}{|c|}{$\begin{array}{l}20.13 \\
\text { Galil }\end{array}$}} & \multicolumn{2}{|l|}{$\mathrm{FOV}\left({ }^{\circ}\right)$} & 10.2 \\
\hline & & & in telescope & & \\
\hline \multicolumn{3}{|c|}{ Objective } & \multicolumn{3}{|c|}{ Eyepiece } \\
\hline \multirow{2}{*}{$\begin{array}{l}\text { Focal length } \\
\qquad(\mathrm{mm})\end{array}$} & $25 \times$ & 80 & \multirow{2}{*}{ Focal length (mm) } & $25 \times$ & -50 \\
\hline & $40 \times$ & 50 & & $40 \times$ & -20 \\
\hline \multirow{2}{*}{ F-number } & $25 \times$ & 4 & \multirow{2}{*}{ F-number } & $25 x$ & 4 \\
\hline & $40 \times$ & 2.5 & & $40 \times$ & 2.5 \\
\hline \multirow{2}{*}{$\mathrm{FOV}\left({ }^{\circ}\right)$} & $25 \times$ & 5.08 & \multirow{2}{*}{ FOV $\left(^{\circ}\right)$} & $25 \times$ & 8.13 \\
\hline & $40 \times$ & 3.25 & & $40 \times$ & 8.13 \\
\hline
\end{tabular}

For initial structure selection, it is of interest to consider not only design parameter conditions, but also the cost and technology of fabrication. As shown in Figure 2a, a doublet-singlet structure was selected as the initial structure for the front objective, which can offer better correction with spherical aberration, coma, and chromatic aberration. A three-component objective was selected for the initial structure of the photographic lens, which consists of four lenses with singlet-singlet-doublet lens configuration (Figure 2b). As we all know, Galilean telescopes constitute positive and negative optical focus lenses. In this design, doublets were selected for both the objective and eyepiece of the Galilean telescopes, which are shown in Figure 2c,d.

(a)

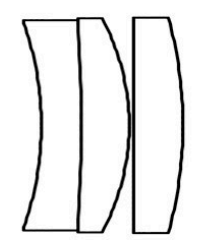

(c)

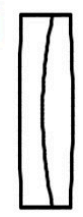

(b)

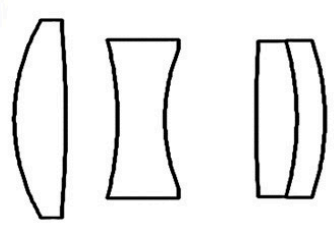

(d)

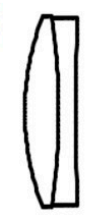

$\prod$

Figure 2. Initial structure of the optical system. (a) Structure of the doublet-singlet lens for the front objective. (b) Three-component objective as the initial structure of the photographic lens. (c,d) The initial structures of Galilean telescopes with $25 \times$ and $40 \times$ magnification, respectively.

\subsection{Optimization}

As described in the previous section, the initial structures of the three subsystems were determined. Then, optimization must be undertaken to correct aberration. We monitored and optimized the imaging quality of each magnification by using multiconfigurations in Zemax software. In consideration of the fact that the DSLM is mainly used for ophthalmic examination, and the medical light source wavelength range is 560-610 $\mathrm{nm}$ [24], we selected the default visible wavelength band in Zemax. In the optimization, the three wavelengths of $486 \mathrm{~nm}$ (blue hydrogen F line), $588 \mathrm{~nm}$ (yellow sodium D line), and $656 \mathrm{~nm}$ (red hydrogen $C$ line) were weighted and optimized during the design process. In Zemax, the RAED operand (real ray angle of exitance) can be adopted to make sure the incident and emergent light of the Galilean telescope are parallel. The EFLY operand (effective focal length in lens 
units) was applied to control the focal length of each subsystem. In addition, center thickness and edge thickness were also constrained to avoid overlap between lenses. The refractive index and Abbe number of glass were constrained to ensure reasonable material. The CDGM (CDGM GLASS CO., Ltd., Chengdu, Sichuan, China) glass catalogs were selected.

Because the DSLM is a non-coaxial system, a progressive optimization strategy was used. In the first step of the optimization, a good optimization result was achieved in the original coaxial system. In the second step, the decentration of the front objective was enlarged gradually during the optimization. The maximum value of decentration is 10.4. It should be noted that, along the $y$-axis, the object point is no longer symmetrically distributed with regard to the optical axis, $\mathrm{M}$. In order to accurate evaluate the imaging quality of the optical system, the actual light needs to be tracked from different field points. As shown in Figure 1, multiple field points in both the $\pm Y$ and $+X$ directions are required to correct the aberrations. The field points were placed at $(0,0) \mathrm{mm},(0,2.25) \mathrm{mm},(0,-2.25) \mathrm{mm},(0,-3.15) \mathrm{mm}$, $(2.25,0) \mathrm{mm}$, and $(0,4.5) \mathrm{mm}$ to describe the imaging quality of the entire optical system. Moreover, coma and astigmatism must be corrected firstly; these affect the image clarity directly. The complex operands, including TRAY (transverse aberration Y direction measured in the image plane with respect to the chief ray), SUMM (sum of two operands), and so on, are taken to control coma and astigmatism. From the optimization results, the high-magnification image quality in the sagittal direction is far better than that in the meridian direction when the decentration displacement is $10.4 \mathrm{~mm}$. In order to correct astigmatism, the image quality of the two directions (meridian and sagittal) should be balanced, and the image quality in the sagittal direction can be reduced to improve the image quality in the meridian direction.

\section{Design Results}

The layout of the non-coaxial DSLM optical system consisted of 11 lens elements with spherical surfaces. Lenses with spherical surfaces and in the least number were employed in the design to reduce cost and assembly difficulty. As shown in Figure 3a, the elements E1 and E2 comprise the front objective; E3 and E4 comprise the Galilean telescope; and E5, E6, and E7 comprise the photographic lens. In the design, the beam splitter is replaced with a parallel plate. The structures of the Galilean telescope, as illustrated in Figure $3 \mathrm{a}, \mathrm{b}$, in which the eyepiece is before the objective lens, can provide magnifications of $6 \times$ and $10 \times$. The magnifications of $25 \times$ and $40 \times$ can be provided as shown in Figure $3 \mathrm{~d}$,e, where the objective is before the eyepiece lens. It can be seen in Figure $3 \mathrm{c}$ that the Galilean telescope can be removed from the light path, and the magnification of this system is $16 \times$. The designed lens prescription is provided in Table 2.
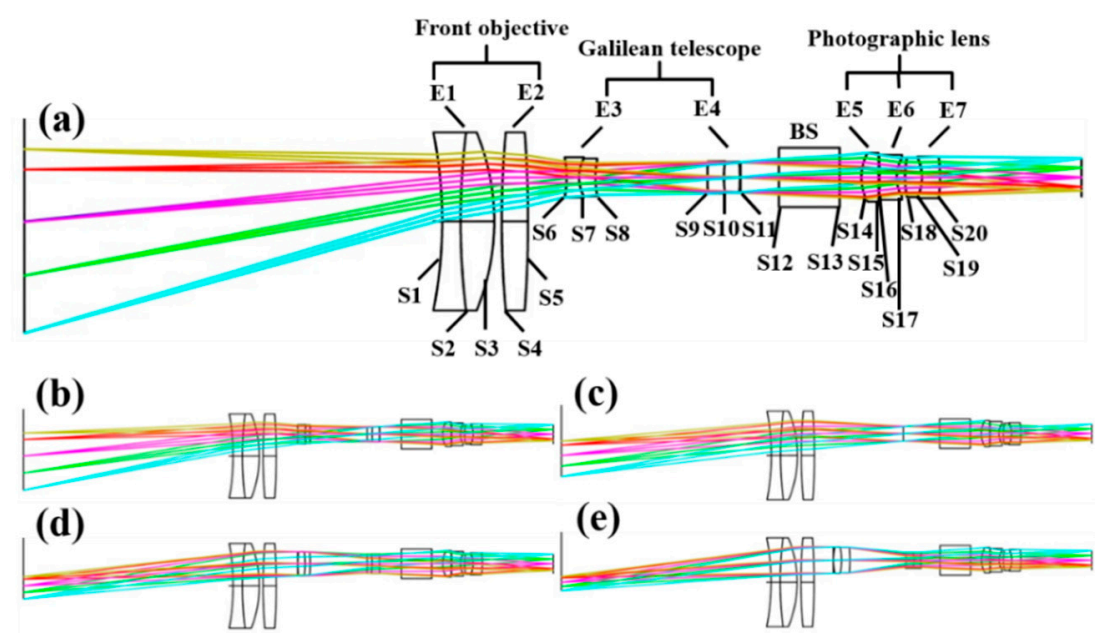

(c)

(e)

Figure 3. Layout of the non-coaxial DSLM optical system. The magnifications of the system are (a) $6 \times$, (b) $10 \times$, (c) $16 \times$, (d) $25 \times$, and (e) $40 \times$. BS: Beam Splitter. 
Table 2. Prescription for the DSLM system.

\begin{tabular}{|c|c|c|c|c|c|c|c|c|c|}
\hline \multirow{2}{*}{ Surface } & \multicolumn{3}{|c|}{ Radius } & \multicolumn{3}{|c|}{ Thickness } & \multicolumn{3}{|c|}{ Glass } \\
\hline & $16 \times$ & $25 \times$ & $40 \times$ & $16 \times$ & $25 \times$ & $40 \times$ & $16 \times$ & $25 \times$ & $40 \times$ \\
\hline 1 & & -110.90 & & & 4.24 & & & $(1.72,29.5)$ & \\
\hline 2 & & 174.75 & & & 7.75 & & & $(1.61,58.6)$ & \\
\hline 3 & & -56.4 & & & 1.71 & & & & \\
\hline 4 & & 240.58 & & & 6 & & & $(1.61,58.6)$ & \\
\hline 5 & & -297.07 & & $57.38 * 1$ & $9.88 * 2$ & $9.88 * 3$ & & & \\
\hline 6 & & 58.08 & 31.92 & & 3.36 & 3.89 & & $\begin{array}{l}(1.47, \\
66.8)\end{array}$ & $\begin{array}{l}(1.52, \\
56.8)\end{array}$ \\
\hline 7 & & -33.99 & -29 & & 2.4 & 3.95 & & $\begin{array}{c}(1.69) \\
30)\end{array}$ & $\begin{array}{l}(1.80, \\
25.5)\end{array}$ \\
\hline 8 & & -62.92 & -56.77 & & 27.81 & 25.74 & & & \\
\hline 9 & & -75.58 & -21.39 & & 2.26 & 3.4 & & $\begin{array}{l}(1.68, \\
31.2)\end{array}$ & $\begin{array}{c}(1.70, \\
30)\end{array}$ \\
\hline 10 & & -16.28 & -9.58 & & 3.32 & 2.7 & & $\begin{array}{l}(1.66, \\
48.4)\end{array}$ & $\begin{array}{l}(1.61, \\
55,8)\end{array}$ \\
\hline 11 & & 54.73 & 25.08 & & 8.35 & 7.82 & & & \\
\hline 12 & & Infinity & & & 14 & & & $(1.52,64.2)$ & \\
\hline 13 & & Infinity & & & 5 & & & & \\
\hline 14 & & 12.78 & & & 3.91 & & & $(1.73,54.7)$ & \\
\hline 15 & & 723.16 & & & 0.26 & & & & \\
\hline 16 & & -110.46 & & & 3.94 & & & $(1.62,36.6)$ & \\
\hline 17 & & 9.49 & & & 1.93 & & & & \\
\hline 18 & & 28.56 & & & 3.84 & & & $(1.53,60.5)$ & \\
\hline 19 & & -10.38 & & & 4.3 & & & $(1.64,60.2)$ & \\
\hline 20 & & -42.66 & & & & & & & \\
\hline
\end{tabular}

In the actual optical system, its point image becomes a speckle because of diffraction of light and aberrations. In Zemax, the characteristics of imaging faculae can be observed through spot diagrams, which are representations of the imaging quality. Figure 4 shows the spot diagrams for the different magnifications of $6 \times, 10 \times, 16 \times, 25 \times$, and $40 \times$. Three representative wavelengths of $486 \mathrm{~nm}, 588 \mathrm{~nm}$, and $656 \mathrm{~nm}$ were analyzed, which are indicated by different colors. As we all know, the spatial resolution of traditional microscopy is limited by the diffraction limit, and the minimum resolvable detail can be scaled by the radius of the Airy disc [25]. At magnifications of $6 \times, 10 \times, 16 \times, 25 \times$, and 40x, the radii of the Airy disc (dark circle) were $5.018 \mu \mathrm{m}, 5.017 \mu \mathrm{m}, 5.018 \mu \mathrm{m}, 5.014 \mu \mathrm{m}$, and $7.164 \mu \mathrm{m}$, respectively. In Figure $4 a-c$, it can be seen that the spots of the $6 \times, 10 \times$, and $16 \times$ magnifications at different fields are almost within the Airy disc. In the systems with high magnification (25× and 40×), as shown in Figure 4d,e, the spot size at $656 \mathrm{~nm}$ is slightly larger than the Airy disc due to the chromatic focal shift. The maximum root-mean-square (RMS) radii of diffuse spots were $2.834 \mu \mathrm{m}, 3.463 \mu \mathrm{m}$, $3.590 \mu \mathrm{m}, 3.658 \mu \mathrm{m}$, and $5.459 \mu \mathrm{m}$ for the magnifications of $6 \times, 10 \times, 16 \times, 25 \times$, and $40 \times$, respectively. On the whole, all the spots are well within the Airy disk, which indicates that quasi-diffraction-limited performance was achieved for all the wavelengths, all the fields, and all the magnifications in the designed system.

The modulation transfer function (MTF) can comprehensively reflect the imaging quality of an optical system and is one of the most important evaluation methods for optical design [26]. In this study, since the pixel size of the sensor is $\sigma=2.2 \mu \mathrm{m}$, the Nyquist sampling spatial frequency of the CCD can be calculated as $N s=1 / 2 \sigma=227 \mathrm{lp} / \mathrm{mm}$. In the full-color image plane, the cut-off frequency of the system was set to $\mathrm{Ns} / 2=113.5 \mathrm{lp} / \mathrm{mm}$. Figure $5 \mathrm{a}-\mathrm{e}$ shows the polychromatic diffraction MTF curves for each configuration with different magnifications and across the entire fields. The MTFs for all fields at $115 \mathrm{lp} / \mathrm{mm}$ are greater than 0.3 (for magnification of $6 \times$ ), 0.2 (for magnifications of $10 \times$, $16 \times$ and $25 \times$ ), or 0.15 (for magnification of $40 \times$ ). The MTF curves for all fields are all close to the 
diffraction-limited (dark line) cases, which clearly demonstrates that this DSLM optical system enables high-quality imaging performance over a broad range of spatial frequencies in these configurations.

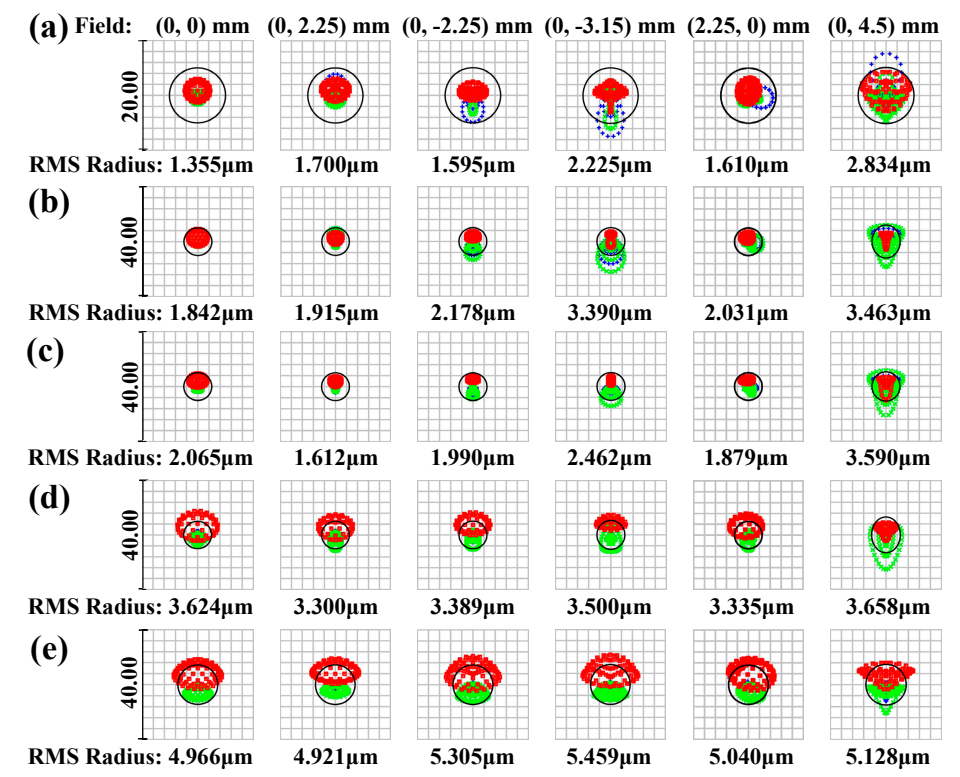

Figure 4. Spot diagrams of different magnifications: (a) 6×, (b) 10×, (c) 16×, (d) 25x, and (e) 40×. RMS: root-mean-square.

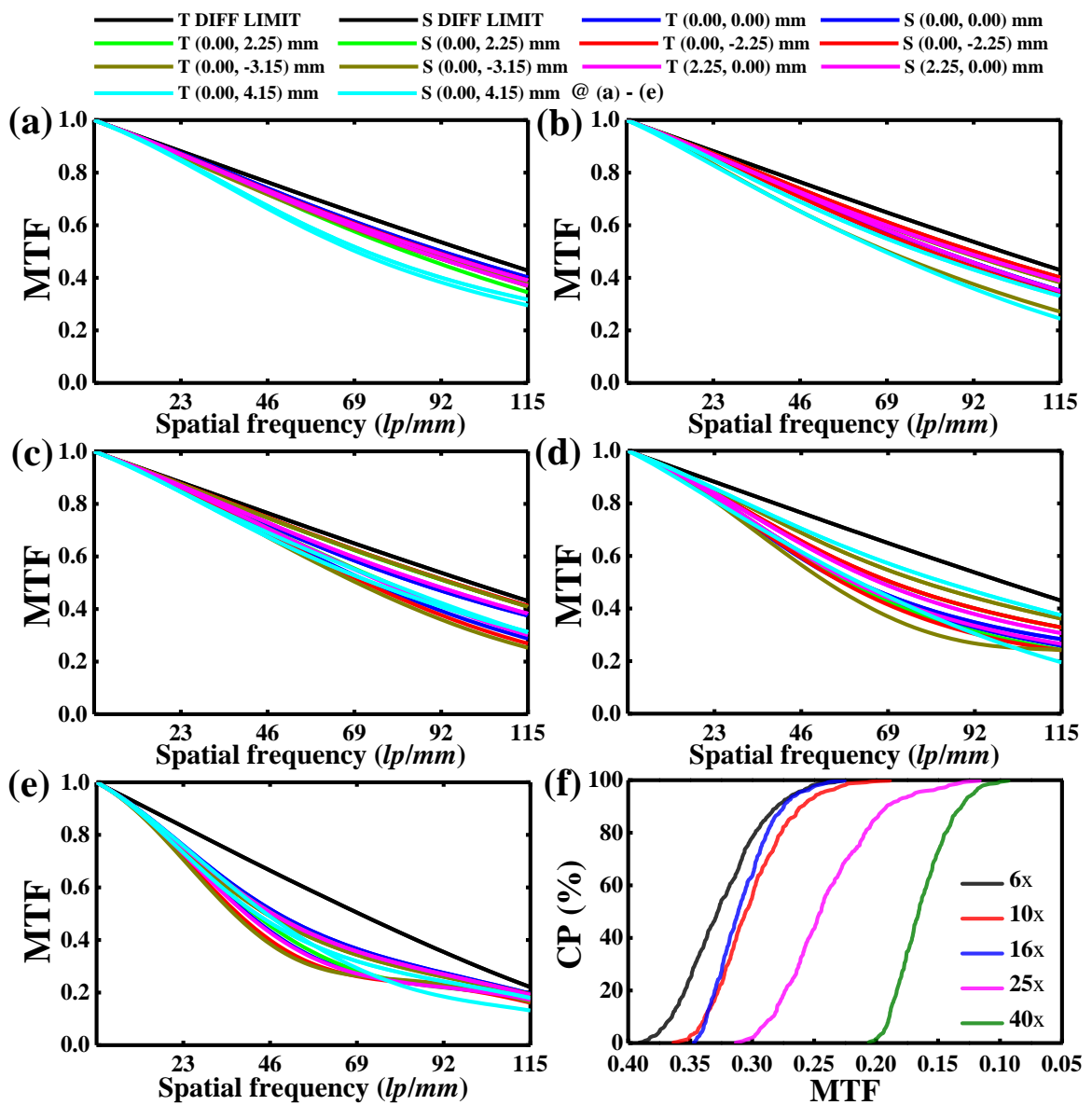

Figure 5. (a-e) Polychromatic diffraction modulation transfer function (MTF) curves of different magnifications: (a) $6 \times$, (b) $10 \times$, (c) $16 \times$, (d) $25 \times$, and (e) 40×. (f) The cumulative probability (CP) functions of samples dependent on the MTFs with different magnifications. 


\section{Tolerance Analysis of the Optical System}

The design results indicated that the DSLM system has excellent imaging quality. However, in the actual production process, irrational distributions of manufacturing and assembly tolerances will cause degradation of the system performance and imaging quality $[27,28]$. In this section, we present the results of tolerance analysis using MTF errors performed in Zemax. Realistic manufacturing and alignment tolerances were applied to evaluate the as-built performance of the DSLM system. As shown in Figure 3a, the surfaces of the optical structure were numbered from S1 to S20. The 500 Monte Carlo samples were run to calculate the MTF at $115 \mathrm{lp} / \mathrm{mm}$ and five worst offenders are shown in Figure 6. Among the worst offenders, variations in thickness and decentration of the E5 and E6 elements (S14-S17) were more sensitive at 6-25×. Variations in the Abbe number of E1 (S1-S3) and decentration of the S7, S8, and S10 surfaces were more sensitive at 40×. Therefore, variations in center thickness, surface/element decentration of the Galilean telescope and photographic lens, and the Abbe number of the front objective were more sensitive in this design, and a higher precision level can be applied to these elements. Other elements can be manufactured with different, lower precision levels and looser tolerances. To select the appropriate tolerances for the system, tolerances were adjusted repeatedly according to the sensitivity analysis result. The final tolerance values are shown in the Table 3.

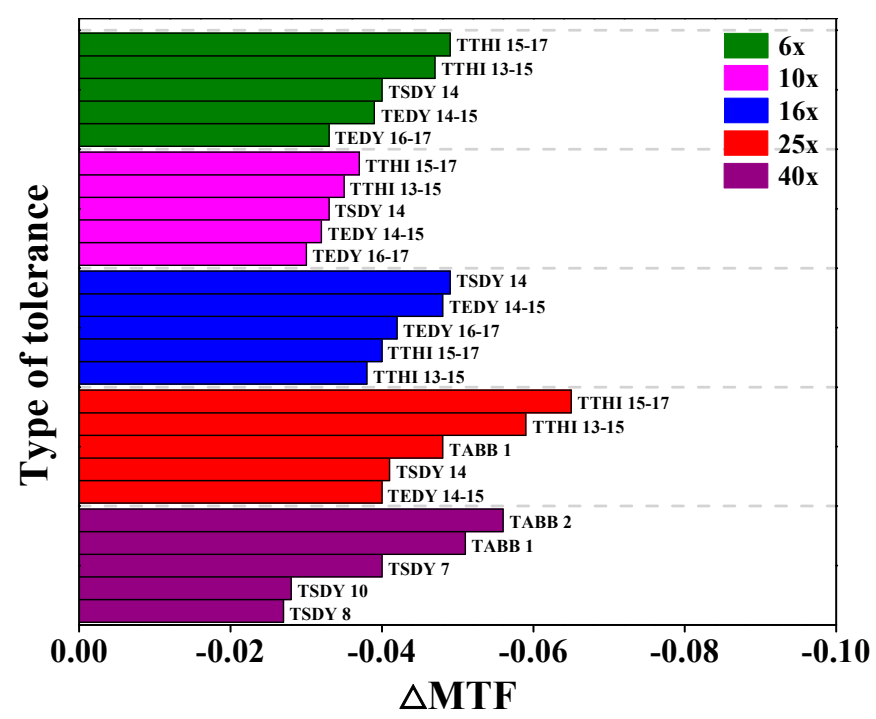

Figure 6. Worst offenders list for tolerance analysis. $\triangle \mathrm{MTF}$ : the variation of MTF, where a negative value indicates a decrease. TTHI: tolerance for thickness or position in lens units. TSDX(Y): tolerance for standard surface $X(Y)$ decentration in lens units. TEDX $(Y)$ : tolerance for element $X(Y)$ decentration in lens units. TIRR: tolerance for standard surface irregularity. TABB: tolerance for Abbe number. The number after the type is the surface number as shown in Figure 3a.

Table 3. System tolerances.

\begin{tabular}{cccc}
\hline \multirow{2}{*}{ Tolerance } & \multicolumn{3}{c}{ Value } \\
\cline { 2 - 4 } & Front Objective & Galilean Telescope & Photographic Lens \\
\hline Radius (fringes) & \pm 5 & \pm 3 & \pm 3 \\
Irregularity (fringes) & \pm 0.5 & \pm 0.3 & \pm 0.3 \\
Center Thickness (mm) & \pm 0.05 & \pm 0.025 & \pm 0.025 \\
Surface/Element & \pm 0.02 & \pm 0.02 & \pm 0.01 \\
Decenter (mm) & \pm 1 & \pm 1 & \pm 1 \\
Surface/Element Tilt (') & \pm 0.001 & \pm 0.001 & \pm 0.001 \\
Refractive Index & \pm 0.025 & \pm 0.025 & \pm 0.025 \\
Space Thickness (mm) & $0.1 \%$ & $0.1 \%$ & $0.1 \%$ \\
Abbe & & &
\end{tabular}


For other aspects considered, the cumulative probability (CP) functions of samples dependent on the MTFs with different magnifications are shown in Figure 5f. The MTFs of more than 90 percent of the samples were greater than $0.28,0.26,0.27,0.19$, and 0.13 at magnifications of $6 \times, 10 \times, 16 \times, 25 \times$, and $40 \times$, respectively. The MTF of $40 \times$ is small due to the nominal criterion of the MTF being 0.19 at $115 \mathrm{lp} / \mathrm{mm}$. From experience in tolerance analysis, values greater than 0.1 are acceptable. Although MTFs can be improved with tighter tolerance, the manufacturing is more difficult, with lower yield and greater cost. To reduce the cost of manufacturing and assembly, a balance between the manufacturing and assembly tolerances and the MTF of the system is required. Uniform tolerance replacement of individual values is adopted to avoid complexity in manufacturing. According to [29], which provides 10 classes of tolerance, the manufacturing and assembly tolerances of the designed system finally listed in Table 3 belong to the low-to-middle class, and optics manufacturing is thus easily realized.

\section{Conclusions}

In conclusion, we analyzed and designed a DSLM. The initial structures of the front objective, Galilean telescope system, and photographic lens were solved analytically, and the progressive optimization process of the non-coaxial system was described in detail. The analysis results from spot diagrams and MTFs indicated that quasi-diffraction-limited performance can be achieved in the designed system. The maximum RMS radii of diffuse spots were lower than $5.459 \mu \mathrm{m}$ at $40 \times$ magnification. At the spatial frequency of $115 \mathrm{lp} / \mathrm{mm}$, the MTFs for all fields were above 0.15 in all configurations, which can meet the requirements of CCD imaging. In addition, we gave proper manufacturing and assembly tolerances for the design, which can reduce the cost of manufacturing and assembly and increase productive benefits. The designed high-performance DSLM has good application prospects in the field of optometry. Finally, this design is more about providing a methodology for the design of a digital-zoom microscope in biomedical imaging instruments, rather than only giving a design result.

Author Contributions: Conceptualization, X.C., H.Z. and F.L.; methodology, Y.H. and S.H.; software, Y.H.; data curation, Y.H. and X.C.; writing - original draft preparation, Y.H.; writing-review and editing, Y.H and X.C.; funding acquisition, X.C. and H.Z. All authors have read and agreed to the published version of the manuscript.

Funding: This research was funded by the Natural Science Foundation of China (Grant No. 51607040); Young Scholars Program of the Fujian Education Department of China (Grant Nos. JAT190468; JT180588); Cultivation Fund of Young Scientific Research Talents of Fujian Jiangxia University (Grant No. JXZ2019013); and High-level Talent Plan of Fujian Jiangxia University.

Conflicts of Interest: The authors declare no conflict of interest.

\section{References}

1. Davidovits, P.; Egger, M.D. Scanning Laser Microscope. Nature 1969, 223, 831. [CrossRef]

2. Huang, B.; Wu, M.; Wang, W.; De Camilli, P.; Zhuang, X. Three-dimensional Super-resolution Fluorescence Microscopy and Its Application to Clathrin Mediated Endocytosis. Annu. Rev. Biochem. 2009, 78, 993-1016. [CrossRef]

3. Brasnu, E.; Bourcier, T.; Dupas, B.; Degorge, S.; Rodallec, T.; Laroche, L.; Borderie, V.; Baudouin, C. In vivo confocal microscopy in fungal keratitis. Br. J. Ophthalmol. 2006, 91, 588-591. [CrossRef]

4. Novack, G.; Moyer, E.D. Slit Lamp-Based Ocular Scoring Systems: Commentary. J. Ocul. Pharmacol. Ther. 2018, 34, 237-238. [CrossRef]

5. Kumar, S.; Wang, E.-H.; Pokabla, M.J.; Noecker, R.J. Teleophthalmology Assessment of Diabetic Retinopathy Fundus Images: Smartphone Versus Standard Office Computer Workstation. Telemed. e-Health 2012, 18, 158-162. [CrossRef]

6. Rosolen, S.; Rivière, M.-L.K.; Lavillegrand, S.; Gautier, B.; LeGargasson, J.-F.; Picaud, S. Use of a combined slitlamp SDOCT to obtain anterior and posterior segment images in selected animal species. Veter Ophthalmol. 2012, 15, 105-115. [CrossRef] 
7. Liu, X.; Jiang, J.; Zhang, K.; Long, E.; Cui, J.; Zhu, M.; An, Y.; Zhang, J.; Lin, H.; Lin, Z.; et al. Localization and diagnosis framework for pediatric cataracts based on slit-lamp images using deep features of a convolutional neural network. PLoS ONE 2017, 12, e0168606. [CrossRef]

8. Martin, R. Cornea and anterior eye assessment with slit lamp biomicroscopy, specular microscopy, confocal microscopy, and ultrasound biomicroscopy. Indian J. Ophthalmol. 2018, 66, 195-201.

9. Ahmed, F.; House, R.J.; Feldman, B.H. Corneal Abrasions and Corneal Foreign Bodies. Prim. Care Clin. Off. Pr. 2015, 42, 363-375. [CrossRef]

10. Tang, K.; Le Beck, D.; Weissman, B.K. Shooting a Film Photo Slit Lamp into the Digital Age. Optom. Vis. Sci. 2006, 83, 616-618. [CrossRef]

11. Rossi, G.; Zerbini, G.; Castro, G.C.; Ortega, T.A.; Mota, A.D.; Yasuoka, F.M.M. Zoom Slit Lamp Adapter for Ophthalmological Green Laser. In Proceedings of the World Congress on Medical Physics and Biomedical Engineering 2006; Springer Science and Business Media LLC: New York, NY, USA, 2009; Volume 25, pp. 56-58.

12. Ye, Y.; Jiang, H.; Zhang, H.; Karp, C.L.; Zhong, J.; Tao, A.; Shao, Y.; Wang, J. Resolution of Slit-Lamp Microscopy Photography Using Various Cameras. Eye Contact Lens Sci. Clin. Pr. 2013, 39, 205-213. [CrossRef]

13. Liu, C.-H.; Yen, C.-H.; Wang, G.-Q.; Lin, T.Y. Semi-permanent smartphone adapter for microscopes: Design demonstration and workflow testing using a slit-lamp biomicroscope. Taiwan J. Ophthalmol. 2019, 9, 111-117. [CrossRef]

14. Cassi, I.; Salvatelli, A.; Bizai, G.; Hadad, A.; Arduh, D.R.; Drozdowicz, B. Images Digitization and Characterization of Surface and Fundus obtained through a Slit Lamp Adapted. In Proceedings of the VII Latin American Congress on Biomedical Engineering CLAIB 2016, Santander, Colombia, 26-28 October 2016; Springer Science and Business Media LLC: New York, NY, USA, 2017; Volume 60, pp. 137-140.

15. Thompson, K. Description of the third-order optical aberrations of near-circular pupil optical systems without symmetry. J. Opt. Soc. Am. A 2005, 22, 1389-1401. [CrossRef]

16. Thompson, K.P.; Schmid, T.; Cakmakci, O.; Rolland, J.P. Real-ray-based method for locating individual surface aberration field centers in imaging optical systems without rotational symmetry. J. Opt. Soc. Am. A 2009, 26, 1503-1517. [CrossRef]

17. Wang, J.; Guo, B.; Sun, Q.; Lu, Z. Third-order aberration fields of pupil decentered optical systems. Opt. Express 2012, 20, 11652-11658. [CrossRef]

18. Hopkins, H.H. The Wave Theory of Aberrations; Oxford University Press: Oxford, UK, 1950; pp. 72-95.

19. Zhu, J.; Hou, W.; Zhang, X.; Jin, G. Design of a low F-number freeform off-axis three-mirror system with rectangular field-of-view. J. Opt. 2014, 17, 15605. [CrossRef]

20. Yang, T.Y.T.; Zhu, J.Z.J.; Jin, A.G.J.A.G. Compact freeform off-axis three-mirror imaging system based on the integration of primary and tertiary mirrors on one single surface. Chin. Opt. Lett. 2016, 14, 60801-60805. [CrossRef]

21. Meng, Q.; Wang, W.; Ma, H.; Dong, J. Easy-aligned off-axis three-mirror system with wide field of view using freeform surface based on integration of primary and tertiary mirror. Appl. Opt. 2014, 53, 3028-3034. [CrossRef]

22. Yu, D.-Y.; Tan, H.-Y. Engineering Optics, 4th ed.; Chinese Machine Press: Beijing, China, 2015; pp. $137-178$.

23. Liao, W.; Xiao, Z.; Chen, G. Design of a front objective in a monocular zoom video microscope. SPIE/COS Photonics Asia 2012, 8557, 85571. [CrossRef]

24. Wu, J.; Lou, S.; Xiao, Z.; Geng, L.; Zhang, F.; Wang, W.; Liu, M. Design of optical system for binocular fundus camera. Comput. Assist. Surg. 2017, 22, 61-69. [CrossRef]

25. Lan, G.; Mauger, T.F.; Li, G. Design of high-performance adaptive objective lens with large optical depth scanning range for ultrabroad near infrared microscopic imaging. Biomed. Opt. Express 2015, 6, 3362-3377. [CrossRef] [PubMed]

26. Choi, H.; Ryu, J.-M.; Yeom, J. Development of a Double-Gauss Lens Based Setup for Optoacoustic Applications. Sensors 2017, 17, 496. [CrossRef] [PubMed]

27. Choi, H.; Ryu, J.-M. Design of Wide Angle and Large Aperture Optical System with Inner Focus for Compact System Camera Applications. Appl. Sci. 2019, 10, 179. [CrossRef] 
28. Hu, X.; Hua, H. Design and tolerance of a free-form optical system for an optical see-through multi-focal-plane display. Appl. Opt. 2015, 54, 9990-9999. [CrossRef] [PubMed]

29. Chang, C.-W.; Ho, G.-H.; Wang, C.-L.; Chao, W.-C.; Griffith, J.D. Tolerance analysis of lenses with high zoom ratio. In Proceedings of the International Society for Optical Engineering, Changchun, China, 21-26 August 2006.

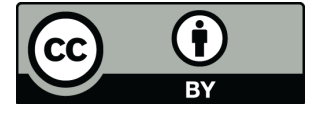

(C) 2020 by the authors. Licensee MDPI, Basel, Switzerland. This article is an open access article distributed under the terms and conditions of the Creative Commons Attribution (CC BY) license (http://creativecommons.org/licenses/by/4.0/). 